\title{
Composto de lodo têxtil em plântulas de soja e trigo
}

\author{
Ademir Sérgio Ferreira de Araújo( $^{(1)}$, Regina Teresa Rosim Monteiro(2) e Patrícia Felippe Cardoso ${ }^{(3)}$
}

\begin{abstract}
(1)Universidade Estadual do Piauí, Campus de Parnaíba, Av. Nossa Senhora de Fátima, CEP 64202-220 Parnaíba, PI. E-mail: asfaruaj@yahoo.com.br (2)Centro de Energia Nuclear na Agricultura, Caixa Postal 96, CEP 13400-970 Piracicaba, SP. E-mail: monteiro@cena.usp.br (3)Escola Superior de Agricultura Luiz de Queiroz, Dep. de Genética, Caixa Postal 83, CEP $13400-970$ Piracicaba, SP. E-mail: pfcardos@esalq.usp.br
\end{abstract}

\begin{abstract}
Resumo - O objetivo deste trabalho foi avaliar o efeito do composto de lodo têxtil sobre plântulas de soja e trigo. O composto de lodo têxtil foi misturado com água e solução nutritiva nas seguintes concentrações (g do composto em $1.000 \mathrm{~mL}$ de água e solução nutritiva): $19 \mathrm{~g} \mathrm{~L}^{-1}, 38 \mathrm{~g} \mathrm{~L}^{-1}, 76 \mathrm{~g} \mathrm{~L}^{-1} \mathrm{e} 152 \mathrm{~g} \mathrm{~L}^{-1}$. Um controle, constituído de água e solução nutritiva, foi incluído no experimento. $\mathrm{O}$ delineamento experimental foi inteiramente casualizado com quatro repetições. As plântulas de soja e trigo foram expostas aos extratos do composto, em sistema de hidroponia com aeração e, após 15 dias, foram avaliadas a massa da matéria seca total, altura da parte aérea, comprimento radicular, conteúdo de clorofila e atividade da peroxidase nas folhas e raízes. Houve diminuição na massa da matéria seca total, altura da parte aérea e comprimento radicular das plântulas de soja e trigo com o aumento da concentração do composto, a partir de $38 \mathrm{~g} \mathrm{~L}^{-1}$. A atividade da peroxidase, das raízes e das folhas das plântulas de soja e trigo, aumentou a partir da concentração de $38 \mathrm{~g} \mathrm{~L}^{-1}$. Concentrações maiores do composto de lodo têxtil afetam, de forma prejudicial, as plântulas de soja e trigo.
\end{abstract}

Termos para indexação: fitotoxicidade, resíduo industrial, peroxidase, clorofila.

\section{Textile sludge compost on soybean and wheat seedlings}

\begin{abstract}
The objective of this work was to evaluate the effect of textile sludge compost on soybean and wheat seedlings. The textile sludge compost was mixed with water plus nutritive solution, in the following concentrations (g of compost in 1,000 mL of water plus nutritive solution): $19 \mathrm{~g} \mathrm{~L}^{-1}, 38 \mathrm{~g} \mathrm{~L}^{-1}, 76 \mathrm{~g} \mathrm{~L}^{-1}$ and $152 \mathrm{~g} \mathrm{~L}^{-1}$. A control, constituted of water plus nutritive solution, was included in the experiment. A completely randomized design was used with four replicates. The seedlings were exposed to the concentrations of compost extract, in hydroponic system, and after 15 days, the total dry mass, height of aerial part, root length, chlorophyll content and peroxidase activity of leaves and roots were evaluated. Seedlings of both crops showed a decrease in the total dry mass, height of aerial part and root length with application of compost in concentrations equal to or above $38 \mathrm{~g} \mathrm{~L}^{-1}$. Peroxidase activity in roots and leaves increased with the concentration of compost equal to or above $38 \mathrm{~g} \mathrm{~L}^{-1}$. Concentrations above $19 \mathrm{~g} \mathrm{~L}^{-1}$ of this textile sludge compost are harmfull to soybean and wheat seedlings.
\end{abstract}

Index terms: phytotoxicity, industrial waste, peroxidase, chlorophyll.

\section{Introdução}

O interesse pela produção de compostos orgânicos a partir de resíduos industriais, tais como lodos, tem crescido como alternativa tanto de redução do volume desse passivo ambiental quanto de obtenção de um produto a ser utilizado em solos agrícolas. O lodo biológico têxtil é de composição variável (Balan \& Monteiro, 2001) e normalmente possui teores elevados de matéria orgânica, N, P e micronutrientes (Martinelli et al., 2002). Além disso, contém corantes com metais pesados e agentes patogênicos. Por causa dessa composição e do risco associado com o uso agrícola direto do lodo, a compostagem vem sendo proposta como uma forma alternativa de tratamento. A compostagem é um processo biológico, no qual vários grupos de microrganismos decompõem o substrato orgânico produzindo água, dióxido de carbono e matéria orgânica maturada (Carvalho, 2002). Este processo pode ser realizado em condições aeróbias ou anaeróbias. Segundo Frassinetti et al. (1990), em condições aeróbias, os microrganismos se desenvolvem e transformam a matéria orgânica, eliminando a toxicidade do resíduo. 
A avaliação de compostos orgânicos industriais para uso agrícola tem levado em consideração apenas o conteúdo de elementos químicos, principalmente metais pesados, que podem estar presentes em concentrações elevadas (Kapanen \& Itavaara, 2001). Estes mesmos autores sugerem que são necessários estudos para avaliar a toxicidade do composto antes de determinar valores-limite para sua aplicação no solo. Segundo Tiquia et al. (1996), a avaliação da toxicidade do composto é um dos mais importantes critérios usados pelas agências ambientais do mundo. A OECD (1984) recomenda testes de fitotoxicidade na germinação de sementes e crescimento vegetal como as técnicas mais comuns na avaliação de compostos e, entre as espécies de plantas, incluem-se monocotiledôneas e dicotiledôneas.

O termo fitotoxicidade está normalmente associado a substâncias potencialmente tóxicas, como metais pesados, que se acumulam nos tecidos da planta, afetando seu crescimento e desenvolvimento (Chang et al., 1992). Por causa dessas características, as plantas são usadas como bioindicadoras de substâncias potencialmente tóxicas (Pandolfini et al., 1997). Hauschild (1993) relata que qualquer mudança nas condições ambientais, principalmente por causa da poluição química, pode ser detectada nas plantas pela produção, acumulação e ativação de compostos na forma de enzimas ou metabólitos em resposta ao estresse ocasionado, assim como pela redução na produção de matéria seca e no crescimento radicular. Fletcher (1990) relata que mudanças fisiológicas e bioquímicas como atividade da peroxidase, respiração, teor de clorofila nas folhas podem ocorrer em plantas expostas a substâncias tóxicas.

O objetivo deste trabalho foi avaliar o efeito do composto de lodo têxtil sobre plântulas de soja e trigo.

\section{Material e Métodos}

O composto utilizado foi obtido a partir da mistura do lodo biológico, coletado de uma industria têxtil do Município de Americana, SP, com um material estruturante (cavaco de madeira) necessário para composição da pilha de compostagem. O processo durou 90 dias e, ao final, foram coletadas 20 subamostras em vários locais da pilha, para formarem uma amostra composta cujas características químicas são apresentadas na Tabela 1.

O composto de lodo têxtil foi misturado com água e solução nutritiva de Hoagland nas seguintes concentrações: $19 \mathrm{~g} \mathrm{~L}^{-1}$ (19 g de composto em $1.000 \mathrm{~mL}$ de água e solução nutritiva); $38 \mathrm{~g} \mathrm{~L}^{-1}$ (38 g de composto em
$1.000 \mathrm{~mL}$ de água e solução nutritiva); $76 \mathrm{~g} \mathrm{~L}^{-1}$ (76 g de composto em $1.000 \mathrm{~mL}$ de água e solução nutritiva); $152 \mathrm{~g} \mathrm{~L}^{-1}$ (152 g de composto em $1.000 \mathrm{~mL}$ de água e solução nutritiva). Um controle, constituído de água e solução nutritiva, foi incluído no experimento. As concentrações utilizadas equivalem a aplicação de 19, 38, 76 e $152 \mathrm{Mg} \mathrm{ha}^{-1}$, admitindo-se a incorporação ao solo até a profundidade de $0,1 \mathrm{~m}$. As quantidades de $\mathrm{N}$ do composto aplicadas correspondem, respectivamente, a uma, duas, quatro e oito vezes a fertilização com $100 \mathrm{~kg} \mathrm{ha}^{-1}$ de N (Cetesb, 1999).

Sementes de soja (cultivar IAC Foscarin) e trigo (cultivar IAC 305) foram pré-germinadas em papel de germinação umedecido em água destilada durante cinco dias em uma sala de germinação sob condições controladas, temperatura de $25^{\circ} \mathrm{C}$ e fotoperíodo de $14 / 10$ horas. Foram utilizados vasos de plásticos de $2 \mathrm{~L}$. Cada vaso recebeu dez plântulas distribuídas em uma placa de isopor (15 mm de espessura) com diâmetro compatível à parte superior do vaso. As plântulas cresceram nesses vasos, durante 15 dias, em sistema de hidroponia em casa de vegetação, temperatura de $20^{\circ} \mathrm{C}$ a $28^{\circ} \mathrm{C}$ e fotoperíodo ambiente, acrescidas dos extratos do composto e com as raízes mantidas sempre aeradas por meio de um compressor de ar. Após esse período, as plântulas foram colhidas e nelas foram avaliados sintomas de toxidez nas folhas, massa da matéria seca total, altura da parte aérea, comprimento radicular, conteúdo de clorofila e atividade da peroxidase das folhas e raízes.

Os sintomas de toxidez foram avaliados por meio de uma escala de notas, variando de 0 a 4 , ou seja: 0 , nenhum sintoma; 1 , pequenos pontos cloróticos nas bor-

Tabela 1. Características químicas do composto de lodo têxtil.

\begin{tabular}{lcc}
\hline Parâmetro & $\begin{array}{c}\text { Composto de } \\
\text { lodo têxtil }\end{array}$ & $\begin{array}{c}\text { Limites máximos de } \\
\text { metais pesados segundo } \\
\text { Cetesb (1999) }\end{array}$ \\
\hline $\mathrm{pH}\left(\mathrm{CaCl}_{2}, 0,01 \mathrm{M}\right)$ & 6,8 & - \\
$\mathrm{N}\left(\mathrm{g} \mathrm{kg}^{-1}\right)$ & 1,3 & - \\
$\mathrm{N}-\mathrm{NH}_{4}\left(\mathrm{~g} \mathrm{~kg}^{-1}\right)$ & 1,6 & - \\
$\mathrm{N}-\mathrm{NO}_{3}\left(\mathrm{~g} \mathrm{~kg}^{-1}\right)$ & 51,5 & - \\
$\mathrm{K}\left(\mathrm{g} \mathrm{kg}^{-1}\right)$ & 5,7 & - \\
$\mathrm{P}\left(\mathrm{g} \mathrm{kg}^{-1}\right)$ & 2,2 & - \\
$\mathrm{Ca}\left(\mathrm{g} \mathrm{kg}^{-1}\right)$ & 13,9 & - \\
$\mathrm{Mg}\left(\mathrm{g} \mathrm{kg}^{-1}\right)$ & 4,3 & 4.300 \\
$\mathrm{Cu}\left(\mathrm{mg} \mathrm{kg}^{-1}\right)$ & 110,8 & 7.500 \\
$\mathrm{Zn}\left(\mathrm{mg} \mathrm{kg}^{-1}\right)$ & 397,0 & 85 \\
$\mathrm{Cd}\left(\mathrm{mg} \mathrm{kg}^{-1}\right)$ & $<0,3$ & 3.000 \\
$\mathrm{Cr}\left(\mathrm{mg} \mathrm{kg}^{-1}\right)$ & 73,9 & 420 \\
$\mathrm{Ni}\left(\mathrm{mg} \mathrm{kg}^{-1}\right)$ & 30,4 & 840 \\
$\mathrm{~Pb}\left(\mathrm{mg} \mathrm{kg}^{-1}\right)$ & 33,4 & 75 \\
$\mathrm{Mo}\left(\mathrm{mg} \mathrm{kg}^{-1}\right)$ & $<4,0$ & - \\
\hline
\end{tabular}

(1)Valores sem limites determinados. 
das das folhas; 2, clorose nas bordas das folhas; 3 , pontos necróticos nas bordas das folhas; e 4 , necrose nas bordas das folhas. A altura da parte aérea e o comprimento radicular foram determinados com régua milimetrada. O conteúdo de clorofila foi determinado com clorofilômetro portátil, modelo Minolta SPAD-502 e as leituras foram feitas em pontos situados entre o meio e dois terços do comprimento da folha, a partir da base, e a $20 \mathrm{~mm}$ de uma das margens da folha.

Amostras de $100 \mathrm{mg}$ da parte aérea e raízes foram maceradas em $\mathrm{N}$ líquido e homogeneizadas em $3 \mathrm{~mL}$ de tampão fosfato de sódio $\left(\mathrm{Na}_{2} \mathrm{HPO}_{4} / \mathrm{NaH}_{2} \mathrm{PO}_{4}\right)$ $0,01 \mathrm{~mol} \mathrm{~L}^{-1}, \mathrm{pH}$ 6,0 e centrifugadas a $20.000 \mathrm{~g}$, por $25 \mathrm{~min}$ a $4^{\circ} \mathrm{C}$. A atividade da peroxidase foi medida pela adição de $100 \mathrm{~mL}$ do sobrenadante em 2,9 mL de tampão fosfato contendo guaiacol e água oxigenada. A leitura de absorbância foi feita em espectrofotômetro a $470 \mathrm{~nm}$ e a atividade enzimática foi expressa em $\mathrm{A}_{470} \mathrm{~min}^{-1} \mathrm{mg}^{-1}$ da amostra fresca.

As plântulas foram secadas em estufa a $65^{\circ} \mathrm{C}$, até atingir massa constante, sendo esta a massa da matéria seca total.

Utilizou-se um delineamento experimental inteiramente casualizado com quatro repetições. Os dados foram submetidos à análise de variância, e as médias comparadas pelo teste de Duncan a 5\% de probabilidade ou análise de regressão.

\section{Resultados e Discussão}

O aumento das concentrações do composto de lodo têxtil, acima de $19 \mathrm{~g} \mathrm{~L}^{-1}$, provocou diminuição significativa na massa da matéria seca total, altura da parte aérea e comprimento radicular das plântulas de soja e trigo (Figura 1). A análise de regressão mostrou que a curva polinomial quadrática foi a que melhor se ajustou aos dados, com $\mathrm{R}^{2}>0,90$. Houve uma redução, em relação ao controle, de $29 \%$ a $34 \%$ na massa da matéria seca total; de $15 \%$ a $36 \%$ na altura da parte aérea e de $28 \%$ a $67 \%$ no comprimento radicular das plântulas de soja com a aplicação de 38, 76 e $152 \mathrm{~g} \mathrm{~L}^{-1}$, respectivamente. Quanto às plântulas de trigo, houve redução, em relação ao controle, de $10 \%$ a $40 \%$ na massa da matéria seca total; de $5 \%$ a $17 \%$ na altura da parte aérea; e de $40 \%$ a $54 \%$ no comprimento radicular com a aplicação de 38, 76 e $152 \mathrm{~g} \mathrm{~L}^{-1}$, respectivamente. A aplicação de $19 \mathrm{~g} \mathrm{~L}^{-1}$ do composto não apresentou efeitos negativos nas variáveis avaliadas, em relação ao controle.
A diminuição na massa da matéria seca total é um indicativo do potencial efeito adverso de substâncias às plantas (Kapustka, 1997) e indica que, provavelmente, o aumento das concentrações provocou toxidez por metais pesados, principalmente $\mathrm{Cu}$ e $\mathrm{Zn}$, que se encon-
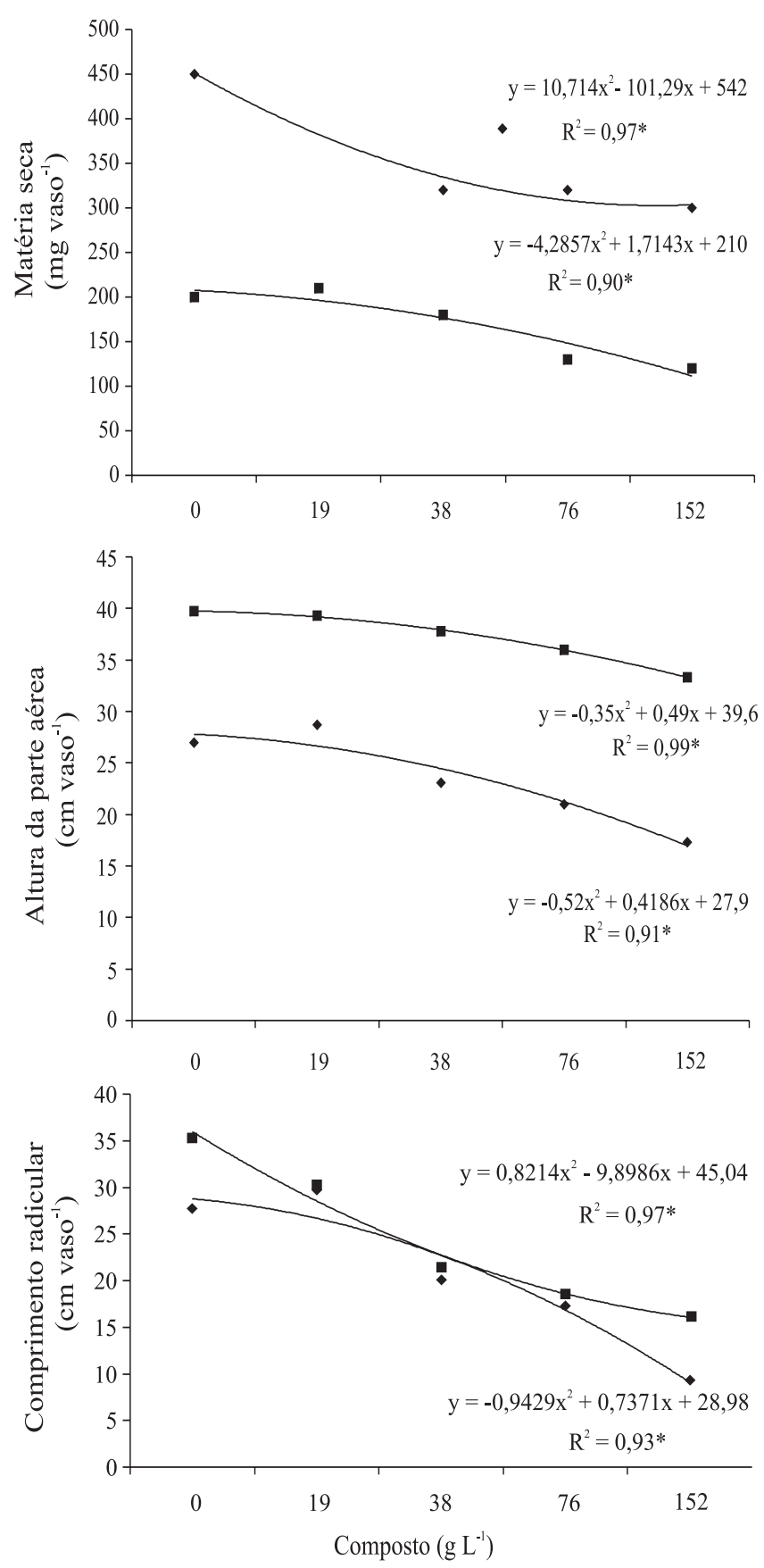

Figura 1. Matéria seca total, altura da parte aérea e comprimento radicular de plântulas de soja ( $>$ ) e trigo ( $\square$ ) submetidas a concentrações crescentes do composto de lodo têxtil. 
tram em maiores quantidades no composto (Tabela 1), embora o composto de lodo têxtil utilizado apresente níveis de metais pesados inferiores aos limites máximos recomendado pela Cetesb (1999). A redução no crescimento é o principal sintoma de toxidez apresentado pelas plantas (Marques et al., 2002). Na concentração de $38 \mathrm{~g} \mathrm{~L}^{-1}$ (taxa de $38 \mathrm{Mg} \mathrm{ha}^{-1}$ ), os níveis de $\mathrm{Cu}$ e Zn na solução foram equivalentes, respectivamente, a 4,2 e $15 \mathrm{mg} \mathrm{L}^{-1}$, considerando a concentração destes elementos no composto e a taxa de aplicação. Estes níveis estão muito abaixo dos descritos por Wallace \& Wallace (1994) para o surgimento de sintomas de toxidez por $\mathrm{Cu}$ e Zn (20 e $80 \mathrm{mg} \mathrm{kg}^{-1}$, respectivamente) em plantas ornamentais cultivadas em solos adubados com lodo de esgoto. Para as plantas, a biodisponibilidade de metais pesados é significativamente diminuída no solo, por causa da interação desses elementos com os colóides do solo (McBride et al., 1998). No presente trabalho, a biodisponibilidade dos metais para as plantas foi máxima, uma vez que os mesmos estavam em solução e, desta forma, os níveis tóxicos são significativamente mais baixos. Ali et al. (2004), comparando um solo artificial e outro natural, observaram que os níveis de toxidez por metais, para cevada, no solo artificial foram mais baixos e concluíram que, nestas condições, existe a máxima biodisponibilidade dos metais.

O crescimento radicular foi mais sensível que a parte aérea às altas concentrações do composto. Houve um declínio significativo na relação raiz/parte aérea das plântulas de soja e trigo em concentrações iguais ou superiores a $38 \mathrm{~g} \mathrm{~L}^{-1}$ (Tabela 2). Ali et al. (2004) também observaram que as raízes são mais sensíveis que a parte aérea à presença de metais pesados. Normalmente a toxicidade dos metais inicia-se na raiz, que é o principal órgão da planta tanto na absorção quanto no acúmulo desses elementos (Marques et al., 2002). Desta forma, a aplicação do composto afetou mais o sistema radicular

Tabela 2. Relação raiz/parte aérea de plântulas de soja e trigo submetidas a concentrações crescentes do composto de lodo têxtil(1).

\begin{tabular}{cll}
\hline Composto $\left(\mathrm{g} \mathrm{L}^{-1}\right)$ & Soja & Trigo \\
\hline 0 & $1,02 \mathrm{a}$ & $0,89 \mathrm{a}$ \\
19 & $1,03 \mathrm{a}$ & $0,77 \mathrm{a}$ \\
38 & $0,87 \mathrm{~b}$ & $0,56 \mathrm{~b}$ \\
76 & $0,82 \mathrm{~b}$ & $0,51 \mathrm{~b}$ \\
152 & $0,53 \mathrm{c}$ & $0,48 \mathrm{~b}$ \\
\hline $\mathrm{CV}(\%)$ & 9,1 & 5,8 \\
\hline
\end{tabular}

(1)Médias seguidas da mesma letra, na coluna, não diferem entre si a 5\% de probabilidade pelo teste de Duncan. do que a parte aérea, e conseqüentemente a relação raiz/parte aérea é fortemente diminuída (Oncel et al., 2000). A inibição do crescimento radicular é um dos principais bioindicadores para estudos de fitotoxicidade de compostos orgânicos (Campbel et al., 1995).

As plântulas de soja e trigo apresentaram uma significativa diminuição no conteúdo de clorofila a partir de $76 \mathrm{~g} \mathrm{~L}^{-1}$ (Tabela 3). Por sua vez, em concentrações a partir de $38 \mathrm{~g} \mathrm{~L}^{-1}$, observaram-se sintomas de toxidez nas folhas, ou seja, pequenos pontos cloróticos nas bordas até o início da necrose foliar. Powell et al. (1996) observaram uma redução no conteúdo de clorofila em macrófitas aquáticas cujas raízes foram expostas a substâncias potencialmente tóxicas e demonstraram uma relação direta com a dose aplicada. Além disso, Weinstein et al. (1990) relataram que plantas de gladíolo expostas a essas condições apresentaram numerosos sintomas visuais, como lesões e mudanças de pigmentação, sendo a deficiência de clorofila reconhecida pela coloração pálida da folha (clorose). A diminuição do conteúdo de clorofila ocasiona simultaneamente um decréscimo na fotossíntese líquida (Oncel et al., 2000) e, conseqüentemente, o crescimento das plantas é menor.

A atividade da peroxidase, tanto nas raízes como nas folhas da soja e do trigo, aumentou com a aplicação das concentrações crescentes do composto de lodo têxtil, acima de $19 \mathrm{~g} \mathrm{~L}^{-1}$ (Figura 2). Este aumento da atividade da peroxidase relacionado com as concentrações do composto é um indicativo de condições adversas no crescimento das plantas, uma vez que a enzima está relacionada ao metabolismo do estresse (Marques et al., 2002)

Tabela 3. Conteúdo de clorofila e surgimento de sintomas de toxidez em folhas de plântulas de soja e trigo submetidas a concentrações crescentes do composto de lodo têxtil.

\begin{tabular}{cccccc}
\hline Composto $\left(\mathrm{g} \mathrm{L}^{-1}\right)$ & \multicolumn{2}{c}{ Leitura do clorofilometro } & & \multicolumn{2}{c}{ Sintomas de toxidez $^{(2)}$} \\
\cline { 2 - 3 } & Soja & Trigo & & Soja & Trigo \\
\hline 0 & $36,7 \mathrm{a}$ & $32,7 \mathrm{a}$ & & 0 & 0 \\
19 & $35,1 \mathrm{a}$ & $32,3 \mathrm{a}$ & & 0 & 0 \\
38 & $32,1 \mathrm{ab}$ & $30,8 \mathrm{ab}$ & & 1 \\
76 & $27,8 \mathrm{~b}$ & $30,6 \mathrm{~b}$ & 2 & 1 \\
152 & $25,2 \mathrm{c}$ & $27,1 \mathrm{c}$ & 3 & 3 \\
\hline $\mathrm{CV}(\%)$ & 2,09 & 4,07 & - & - \\
\hline
\end{tabular}

(1)Médias seguidas da mesma letra, na coluna, não diferem entre si a 5\% de probabilidade pelo teste de Duncan. ${ }^{(2)}$ Escala de notas dos sintomas de toxidez: 0, nenhum sintoma de toxidez; 1, pequenos pontos cloróticos nas bordas das folhas; 2, clorose nas bordas das folhas; 3 , pequenos pontos necróticos nas bordas das folhas; 4 , necrose nas bordas das folhas. 
e funciona como uma barreira protetora contra o efeito prejudicial pela peroxidação de substâncias tóxicas presentes no meio (Dhindsa \& Matawe, 1981).

A atividade da peroxidase nas raízes foi mais influenciada pelas concentrações do composto do que na parte aérea, como pode ser verificado pelo maior aumento da atividade enzimática nas doses mais elevadas do composto (Figura 2). Este aumento na atividade da peroxidase, correlacionado com a diminuição mais acentuada das raízes, sugere a participação desta enzima na redução do crescimento radicular observado nas concentrações mais elevadas. Segundo Byl et al. (1994), a
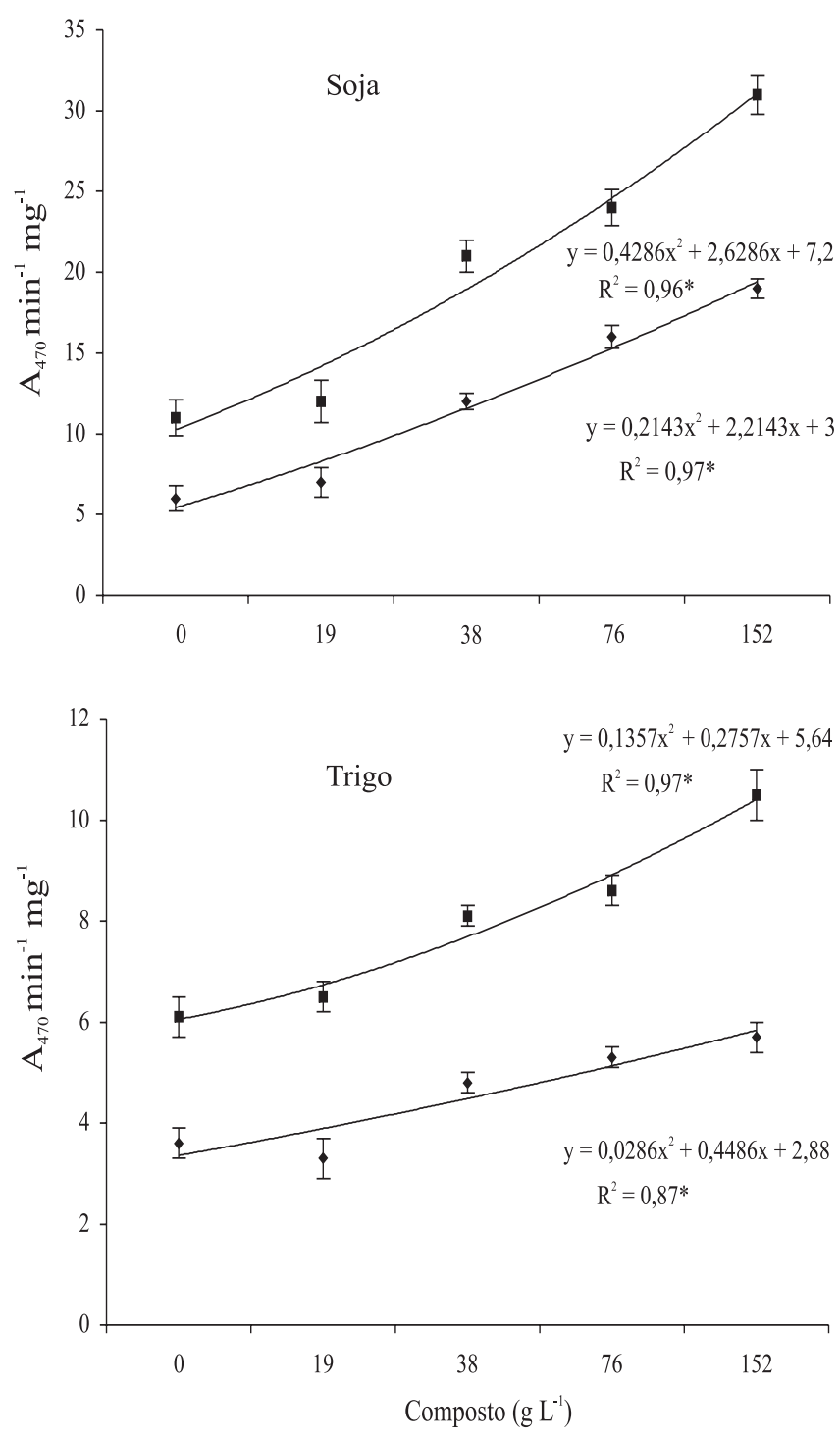

Figura 2. Atividade da peroxidase nas folhas ( ) e nas raízes (ם) de plântulas de soja e trigo submetidas a concentrações crescentes de extrato do composto de lodo têxtil. peroxidase está envolvida no crescimento celular da planta e o aumento da sua atividade ocasiona uma redução no crescimento vegetativo. Correlação significativa entre a atividade da peroxidase e o crescimento radicular também foi encontrada por Pires et al. (2001).

\section{Conclusões}

1. O aumento das concentrações do composto de lodo têxtil afeta, de forma prejudicial, as plântulas de soja e trigo.

2. O comprimento radicular e a atividade da peroxidase são as variáveis mais sensíveis à aplicação do composto de lodo têxtil.

\section{Referências}

ALI, N.A.; ATER, M.; SUNAHARA, G.I.; ROBIDOUX, P.Y. Phytotoxicity and bioacumulation of copper and chromium using barley (Hordeum vulgare L.) in spiked artificial and natural forest soils. Ecotoxicology and Environmental Safety, v.57, p.363-374, 2004.

BALAN, D.S.L.; MONTEIRO, R.T.R. Decolorization of textile indigo dye by lignolytic fungi. Journal of Biotechnology, v.89, p.141-145, 2001.

BYL, T.D.; SUTTON, H.D.; KLAINE, S.J. Evaluation of peroxidase as a biochemical indicator of toxic chemical exposure in the aquatic plant Hydrilla verticillata, Royle. Environmental Toxicology Chemistry, v.13, p.509-515, 1994.

CAMPBEL, A.G.; ZHANG, X.; TRIPEPI, R.R. Composting and evaluating a pulp and paper sludge for use as a soil amendment/ mulch. Compost Science and Utilization, v.84, p.84-95, 1995.

CARVALHO, P.C.T. Compostagem. In: TSUTIYA, M.T.; CAMPARINI, J.B.; ALEM SOBRINHO, P.; HESPANOL, I.; CARVALHO, P.C.T.; MELFI, A.J.; MELO, W.J.; MARQUES, M.O. (Ed.). Biossólidos na agricultura. 2.ed. São Paulo: ABES, 2002. Cap.6. p.181-208.

CETESB. Aplicação de biossólidos em áreas agrícolas: critérios para projeto e operação. Norma P4230. São Paulo, 1999, 29p.

CHANG, A.C.; GRANTO, T.C.; PAGE, A.L. A methodology for establishing phytotoxicity criteria for chromium, copper, nickel and zinc in agricultural land application of municipal sewage sludges. Environmental quality, v.21, p.521-536, 1992.

DHINDSA, R.S.; MATAWE, W. Drought tolerance in two mosses correlated with defense against lipid peroxidation. Journal of Experimental Botany, v.32, p.79-91, 1981.

FLETCHER, J. Use of algae versus vascular plants to test for chemical toxicity. In: WANG, W.; GORSUCH, J.W.; LOWER, W.R. (Ed.). Plants for toxicity assessment. Philadelphia: ASTM, 1990. p.3339.

FRASSINETTI, S.; CITTERIO, S.; NAPPI, P. Chemical changes in sludge stabilization. BioCycle, v.31, p.50-52, 1990. 
HAUSCHILD, M.Z. Chromium content of leaves reveals chromium (III): Stress of higher plants before sensitive biomarkers. Science in the Total Environment, p.1345-1352, 1993. Supplement.

HOAGLAND, D.; ARNON, D.I. The water culture method for growing plants without soil. Berkeley: California Agriculture Experiment Station, 1950.

KAPANEN, A.; ITAVAARA, M. Ecotoxicity tests for compost applications. Ecotoxicology Environmental Safety, v.49, p.1-16, 2001.

KAPUSTKA, L.A. Selection of phytotoxicity tests for use in ecological risk assesments. In: WANG, W.; GORSUCH, J.W.; HUGHES, D. Plants for environmental studies. New York: CRC Press, 1997. p.516-548.

MARQUES, M.O.; MELO, W.J.; MARQUES, T.A. Metais pesados e o uso de biossólidos na agricultura. In: TSUTIYA, M.T.; CAMPARINI, J.B.; ALEM SOBRINHO, P.; HESPANHOL, I.; CARVALHO, P.C.T.; MELFI, A.J.; MELO, W.J.; MARQUES, M.O. (Ed.). Biossólidos na agricultura. 2.ed. São Paulo: ABES, 2002. Cap.12. p.365-403.

MARTINELLI, U.A.; PERON, A.P.; MARTINS, E.P.; SCHARF, M.; BUDAG, N.; BARCELLOS, I.O. Lodo têxtil: um problema ou uma solução. Química Têxtil, v.69, p.16-23, 2002.

McBRIDE, M.; MARTINEZ, C.E.; SAUVE, S. Copper: II Activity in aged suspensions of geothite and organic matter. Soil Science Society of American Journal, v.62, p.1542-1548, 1998. OECD. OECD guidelines for testing of chemicals: guideline 208, terrestrial plants, growth test. Paris, 1984.
ONCEL, I.; KELES, Y.; USTUN, A.S. Interactive effects of temperature and heavy metal stress on the growth and some biochemical compounds in wheat seedlings. Environmental Pollution, v.107, p.315-320, 2000.

PANDOLFINI, T.; GREMIGNI, P.; GABBRIELLI, R. Biomonitoring of soil health by plants. In: PANKHUST, C.; DOUBE, B.M.; GUPTA, V.V.S.R. (Ed.). Biological indicators of soil health. New York: CAB, 1997. p.325-348.

PIRES, N.M.; SOUZA, I.R.P.; PRATES, H.T.; FARIA, T.C.L.; PEREIRA FILHO, I.A.; MAGALHÃES, P.C. Efeito do extrato aquoso de leucena sobre o desenvolvimento, índice mitótico e atividade da peroxidase em plântulas de milho. Revista Brasileira de Fisiologia Vegetal, v.13, p.55-65, 2001.

POWELL, R.L.; KIMERLE, R.A.; MOSER, E.M. Development of plant bioassay to assess toxicity of chemical stressors to emergent macrophytes. Environmental Toxicology Chemistry, v.15, p.15701576, 1996.

TIQUIA, S.M.; TAM, N.F.Y.; HODGKISS, I.J. Effects of composting on phytotoxicity of spent pig-manure sawdust litter. Environmental Pollution, v.93, p.249-256, 1996.

WALLACE, A.; WALLACE, G.A. A possible flaw in EPA'S 1993 new sludge rule due to heavy metal interactions. Communications in Soil Science and Plant Analysis, v.25, p.129-135, 1994.

WEINSTEIN, L.H.; LAURENCE, J.A.; MANSL, R.H.; WALTI, $\mathrm{K}$. The use of native and cultivated plants as bioindicators and biomonitors of pollution damage. In: WANG, W.; GORSUCH, J.W.; LOWER W.R. (Ed.). Plants for toxicity assessment. Philadelphia: ASTM, 1990. p.117-126.

Recebido em 20 de setembrode 2004 e aprovado em 27 de dezembro de 2004 